\title{
The Existence and Uniqueness of Positive Solutions for a Singular Nonlinear Three-Point Boundary Value Problems
}

\author{
Yao Dong, Baoqiang Yan* \\ School of Mathematical Sciences, Shandong Normal University, Jinan, China \\ Email:983743926@qq.com, *yanbqcn@aliyun.com
}

How to cite this paper: Dong, Y. and Yan, B.Q. (2018) The Existence and Uniqueness of Positive Solutions for a Singular Nonlinear Three-Point Boundary Value Problems. Journal of Applied Mathematics and Physics, 6, 2600-2620. https://doi.org/10.4236/jamp.2018.612217

Received: December 9, 2018

Accepted: December 23, 2018

Published: December 26, 2018

\begin{abstract}
Using the method of lower and upper solutions, we study the following singular nonlinear three-point boundary value problems:

$\left\{\begin{array}{l}-x^{\prime \prime}(t)+K(t) x^{-q}(t)=\lambda x^{p}(t), \quad t \in(0,1), \\ x(0)=0, \quad x(1)=a x(\eta),\end{array}\right.$

$0<\eta<1$ and $\lambda$ is a positive parameter and present the existence, uniqueness, and the dependency on parameters of the positive solutions under various assumptions. Our result improves those in the previous literatures.
\end{abstract}

\section{Keywords}

Three-Point Boundary Value Problem, Positive Solution, Lower and Upper Solutions, Eigenvalue and Eigenfunction

\section{Introduction and Main Results}

In this paper, we consider the three-point boundary value problem

$$
\left\{\begin{array}{l}
-x^{\prime \prime}(t)+K(t) x^{-q}(t)=\lambda x^{p}(t), \quad t \in(0,1), \\
x(0)=0, \quad x(1)=\operatorname{ax}(\eta)
\end{array}\right.
$$

where $K \in C[0,1], \quad 0<a<1, \quad 0<\eta<1$, and $\lambda$ is a positive parameter.

The $m$-point boundary value problem for linear second-order ordinary differential equations was initiated by Ilin and Moiseev [1] [2]. Since then, there are many results on the existence of general nonlinear multi-point boundary value problems, see [3] [4] [5] [6] and their references. For examples, in [6], Rynne studied the $\$ \mathrm{~m} \$$-point boundary value problem 


$$
\left\{\begin{array}{l}
-u^{\prime \prime}=f(u), \quad \text { on }(0,1), \quad u \in R \times X, \\
u(0)=0, \quad u(1)=\sum_{i=1}^{m-2} \alpha_{i} u\left(\eta_{i}\right),
\end{array}\right.
$$

where $m \geq 3, \eta_{i} \in(0,1), \quad \alpha_{i}>0$ with $\sum^{m-2} \alpha_{i}<1$ and presented the existence of the sign changing solutions by Rabinowitz bifurcation theorem. Especially, Rynne ([7]) discussed the three-point boundary value problem

$$
\left\{\begin{array}{l}
-u^{\prime \prime}=f(u)+h, \quad \text { on }(0,1), \\
u(0)=0, \quad u(1)=\alpha u(\eta),
\end{array}\right.
$$

and showed the solvability and non-solvability results from either the half-eigenvalue or the Fucik spectrum approach. As we known, the method of upper and lower solutions is very important for the study of the boundary value problems, see [8]-[18]. Therefore, establishing the method of upper and lower solutions for three-point boundary value problems is necessary and important.

In [19], when $f$ is nondecreasing on $x$, Du and Zhao got the methods of upper and lower solutions of

$$
\left\{\begin{array}{l}
-x^{\prime \prime}(t)=f(t, x(t)), \quad t \in(0,1), \\
x(0)=a x(\eta), \quad x(1)=0
\end{array}\right.
$$

and used iterative techniques to study the existence of positive solutions. And in [3] when $f$ is decreasing on $u, D u$ and Zhao considered the existence and uniqueness of positive solutions of the problem

$$
\begin{cases}-u^{\prime \prime}(t)=f(t, u(t)), & t \in(0,1), \\ u(0)=\sum_{i=1}^{m-2} \alpha_{i} u\left(\eta_{i}\right), & u(1)=0\end{cases}
$$

by constructing lower and upper solutions. Wei ([15]) constructed the method of upper and lower solutions for three-point boundary value problems and gave the sufficient and necessary conditions for the existence of positive solutions of the problem

$$
\left\{\begin{array}{l}
-x^{\prime \prime}(t)=f(t, x(t)), \quad t \in(0,1), \\
x(0)=a x(\eta), \quad x(1)=0 .
\end{array}\right.
$$

On the other hand, singular boundary problems arise in the contexts of chemical heterogeneous catalysts, non-Newtonian fluids and also the theory of heat conduction in electrically conducting materials, see [20]-[25] for a detailed discussion. An interesting result comes from [25], in which, using method of upper and lower solutions, Shi and Yao discussed the following problem

$$
\left\{\begin{array}{l}
-\Delta u+K(x) u^{-q}=\lambda u^{p}, \quad x \in \Omega, \\
u(x)>0, \forall x \in \Omega \\
\left.u\right|_{\partial \Omega}=0
\end{array}\right.
$$

where $K \in C^{2, \beta}(\bar{\Omega}), \quad p, q \in(0,1)$ and $\lambda$ is a positive parameter. Under various appropriate assumptions on $K(x)$, Shi and Yao obtained the existence and 
uniqueness of classical solutions.

Motivated by above works, under various appropriate assumptions on $p, q$ and $K(t)$, we will obtain the existence and uniqueness of positive solution of problem (1.1) for $\lambda$ in different circumstances. In our proof, the upper and lower solutions theorem (see [16]) plays an important role in the paper.

Define

$$
K^{*}=\max _{t \in[0,1]} K(t), K_{*}=\min _{t \in[0,1]} K(t) .
$$

The main results of this paper are stated in the following theorems.

Theorem 1.1. When $K_{*}>0$,

1) If $0<p, q<1$, there exists $\bar{\lambda}>0$ such that the problem (1.1) has at least one $C[0,1]$ positive solution $x_{\lambda}(t)$ for $\lambda>\bar{\lambda}$.

2) For $\lambda>\bar{\lambda}$, (1.1) has a maximal solution $\bar{x}_{\lambda}(t)$ and $\bar{x}_{\lambda}(t)$ is increasing with respect to $\lambda$.

Theorem 1.2. When $K^{*}<0$,

1) If $0<p<1,0<q$, (1.1) has at least one $C[0,1]$ positive solution for all $\lambda>0$.

2) If $0<p, q<1,(1.1)$ has an unique $C^{1}[0,1]$ positive solution $x_{\lambda}(t)$ for all $\lambda>0$.

3) $x_{\lambda}(t)$ in (2) is increasing with respect to $\lambda$.

Theorem 1.3. When $K_{*}<0<K^{*}$,

1) If $0<p, q<1$, there exists a $\lambda_{*}>0$ such that the problem (1.1) has at least one $C[0,1]$ posit-ive solution $x_{\lambda}(t)$ for $\lambda>\lambda_{*}$.

2) For $\lambda>\lambda_{*}, x_{\lambda}(t)$ in (1) is increasing with respect to $\lambda$.

Remark 1.1: Note $K(t)>0$ in Theorem (1.1). This is different from the conditions in [3] [15] [19] because $K(t)<0$ in these references.

Remark 1.2: The unique result in Theorem 1.2 is different from that in [3] because we remove the monotonicity of nonlinearity $f$ in $x$.

Remark 1.3: Note $K(t)$ is sigh-changing in Theorem 1.3. This is different from the conditions in [3] [15] [19] because $K(t)<0$ in these references and is different from conditions in [1] [2] [4] [5] [6] [7] [26] because $f$ is continuous at $x=0$ in these references.

This paper is organised as follows. Some preliminary lemmas are stated and proved in Section 2. And Section 3 is devoted to prove the results.

\section{Preliminaries}

In this section, we first consider the following problem

$$
\left\{\begin{array}{l}
-x^{\prime \prime}(t)=f\left(t, x(t), x^{\prime}(t)\right), \quad t \in(0,1), \\
x(0)=0, x(\eta)=\operatorname{ax}(1),
\end{array}\right.
$$

where $\eta \in(0,1), \quad 0<a<1$ and $f \in[0,1] \times \mathbb{R} \times \mathbb{R}$.

Let $C^{1}[0,1]=\{x:[0,1] \rightarrow \mathbb{R} \mid x(t)$ is differential continuous on $[0,1]\}$ with norm 


$$
\|x\|=\max \left\{|x|_{\infty},\left|x^{\prime}\right|_{\infty}\right\},
$$

where $\left|x^{\prime}\right|_{\infty}=\max _{t \in[0,1]}|x(t)|$. Obviously, $C^{1}[0,1]$ is a Banach space. Now we give the definitions of lower and upper solutions for problem (2.1).

Definition 2.1. A function $\alpha(t)$ is called a lower solution to the problem (2.1), if $\alpha(t) \in C[0,1] \cap C^{2}(0,1)$ and satisfies

$$
\left\{\begin{array}{l}
-\alpha^{\prime \prime}(t) \leq f\left(t, \alpha(t), \alpha^{\prime}(t)\right), \quad t \in(0,1), \\
\alpha(0) \leq 0, \quad \alpha(1) \leq a \alpha(\eta) .
\end{array}\right.
$$

Upper solution is defined by reversing the above inequality signs in problem (2.2).

If there exists a lower solution $\alpha(t)$ and an upper solution $\beta(t)$ to problem (2.1) such that $\alpha(t) \leq \beta(t)$, then $(\alpha(t), \beta(t))$ is called a couple of upper and lower solutions of problem (2.1).

Set $D_{\alpha}^{\beta}=\left\{(t, x) \in(0,1) \times \mathbb{R}^{+}, \alpha(t) \leq x \leq \beta(t), t \in(0,1)\right\}$.

We list a lemma for the eigenvalues and eigenfunctions for the following linear problem

$$
\left\{\begin{array}{l}
-x^{\prime \prime}(t)=\lambda x(t), \quad t \in(0,1), \\
x(0)=0, \quad x(1)=\operatorname{ax}(\eta) .
\end{array}\right.
$$

Lemma 2.1. (see [6]) The spectrum $\sigma(L)$ of problem (2.3) consists of a strictly increasing sequence ofeigenvalues $\lambda_{k}>0, k=1,2, \cdots$, with eigenfuctions $\phi_{k}=\sin \left(\lambda_{k}^{\overline{2}} t\right)$. In addition,

1) $\lim _{k \rightarrow+\infty} \lambda_{k}=+\infty$;

2) $\phi_{k}(t)$ has exact $k-1$ simple zeros in $(0,1), k=2,3, \cdots$ and $\phi_{1}$ is strictly positive on $(0,1)$.

Lemma 2.2. Suppose that $h \in L^{1}(0,1)$. Then, for each $\lambda>0$, the problem

$$
\left\{\begin{array}{l}
-x^{\prime \prime}(t)+\lambda x=h(t), \quad t \in(0,1), \\
x(0)=0, x(\eta)=\alpha x(1)
\end{array}\right.
$$

has an unique solution in $C[0,1]$.

Proof. Assume that $v_{1}(t)$ and $v_{2}(t)$ satisfies that

$$
\left\{\begin{array}{l}
-x^{\prime \prime}(t)+\lambda x=h(t), \quad t \in(0,1), \\
x(0)=0, x^{\prime}(0)=1
\end{array}\right.
$$

and

$$
\left\{\begin{array}{l}
-x^{\prime \prime}(t)+\lambda x=h(t), \quad t \in(0,1), \\
x(1)=0, x^{\prime}(1)=-1
\end{array}\right.
$$

respectively. Define

$$
G(t, s)=\frac{1}{\omega} \begin{cases}v_{2}(t) v_{1}(s), & 0 \leq s \leq t \leq 1 \\ v_{1}(t) v_{1}(s), & 0 \leq t \leq s \leq 1,\end{cases}
$$

and

$$
x(t)=\int_{0}^{1} G(t, s) h(s) d s+\frac{e_{1}(t)}{e_{1}(1)-\alpha e_{1}(\eta)} \alpha \int_{0}^{1} G(\eta, s) h(s) d s, s \in[0,1] .
$$

Then 


$$
\begin{aligned}
-x "(t)+\lambda x(t) & =-\frac{1}{\omega}\left[\int_{0}^{t} v_{2}(t) v_{1}(s) h(s) d s+\int_{t}^{1} v_{1}(t) v_{2}(s) h(s) d s\right] " \\
& -\frac{e_{1}{ }^{\prime}(t)}{e_{1}(1)-\alpha e_{1}(\eta)} \alpha \int_{0}^{1} G(\eta, s) h(s) d s+\lambda x(t) \\
& =-\frac{1}{\omega}\left[v_{2}{ }^{\prime}(t) v_{1}(t)-v_{1}^{\prime}(t) v_{2}(t)\right] h(t) \\
& -\frac{1}{\omega}\left[\lambda \int_{0}^{t} v_{2}(t) v_{1}(s) h(s) d s+\lambda \int_{t}^{1} v_{1}(t) v_{2}(s) h(s) d s\right] \\
& -\frac{\lambda e_{1}(t)}{e_{1}(1)-\alpha e_{1}(\eta)} \alpha \int_{0}^{1} G(\eta, s) h(s) d s+\lambda x(t) \\
& =h(t)-\lambda \frac{1}{\omega}\left[\int_{0}^{t} v_{2}(t) v_{1}(s) h(s) d s+\int_{t}^{1} v_{1}(t) v_{2}(s) h(s) d s\right] \\
& -\lambda \frac{e_{1}(t)}{e_{1}(1)-\alpha e_{1}(\eta)} \alpha \int_{0}^{1} G(\eta, s) h(s) d s+\lambda x(t) \\
& =h(t), \quad t \in(0,1)
\end{aligned}
$$

and

$$
\begin{aligned}
x(1)-\alpha x(\eta) & =\int_{0}^{1} G(1, s) h(s) d s+\frac{e_{1}(1)}{e_{1}(1)-\alpha e_{1}(\eta)} \alpha \int_{0}^{1} G(\eta, s) h(s) d s \\
& -\alpha\left[\int_{0}^{1} G(\eta, s) h(s) d s+\frac{e_{1}(\eta)}{e_{1}(1)-\alpha e_{1}(\eta)} \alpha \int_{0}^{1} G(\eta, s) h(s) d s\right] \\
& =\frac{e_{1}(1)}{e_{1}(1)-\alpha e_{1}(\eta)} \alpha \int_{0}^{1} G(\eta, s) h(s) d s \\
& -\alpha\left[\int_{0}^{1} G(\eta, s) h(s) d s+\frac{e_{1}(\eta)}{e_{1}(1)-\alpha e_{1}(\eta)} \alpha \int_{0}^{1} G(\eta, s) h(s) d s\right] \\
& =0 .
\end{aligned}
$$

Hence, $x(t)$ is a $\mathrm{C}[0,1]$ solution to $\operatorname{problem}(2.4)$. Since $\lambda>0$, Lemma 2.1 guarantees that problem $(2.4)$ has an unique $C[0,1]$ solution. The proof is complete. $\square$

Theorem 2.1. Let $\alpha$ and $\beta \in C([0,1]) \cap C^{1}(0,1)$ be lower and upper solutions of (2.1) such that $\alpha \leq \beta$. Let $\bar{\psi} \in L^{1}[0,1]$ and $\bar{\phi}: \mathbb{R}^{+} \rightarrow \mathbb{R}_{0}^{+}$be a continuous function that satisfies

$$
\int_{0}^{\infty} \frac{1}{\bar{\phi}(s)} d s=+\infty .
$$

Suppose $f: D_{\alpha}^{\beta} \times \mathbb{R} \rightarrow \mathbb{R}$ is an $L^{1}$-Carathéodory-function such that

$$
|f(t, x, v)| \leq \bar{\psi}(t) \bar{\phi}(|v|), \forall(t, x) \in D_{\alpha}^{\beta}, v \in \mathbb{R} .
$$

Then the problem (2.1) has at least one solution $x \in C^{1}[0,1]$ such that for all $t \in[0,1]$,

$$
\alpha(t) \leq x(t) \leq \beta(t) .
$$

Proof. The proof proceeds in five steps.

Step 1. We consider a new modified problem. From (2.5), there is an $R>0$ be large enough so that

$$
\int_{0}^{R} \frac{1}{\bar{\phi}(s)} d s>\|\psi\|_{1} .
$$


And (2.6) guarantees that there is an $\bar{N}(t)$ with $\bar{N} \in L^{1}[0,1]$ such that

$$
|f(t, x, v)| \leq \bar{N}(t), \forall(t, x) \in D_{\alpha}^{\beta},|v| \leq R .
$$

Define then

$$
\chi(t, x)=\left\{\begin{array}{l}
\alpha(t), \quad \text { if } \quad x<\alpha(t), \\
x, \quad \text { if } \quad \alpha(t) \leq x \leq \beta(t), \\
\beta(t), \quad \text { if } \quad x>\beta(t)
\end{array}\right.
$$

and

$$
g(t, x, v)=\max \{\min \{f(t, \chi(t, x), v), \bar{N}(t)\},-\bar{N}(t)\} .
$$

Choose a $\lambda>0$ and consider the new boundary value problem

$$
\left\{\begin{array}{l}
-x^{\prime \prime}(t)+\lambda x=g\left(t, x(t), x^{\prime}(t)\right)+\lambda \chi(t, x(t)), \quad t \in(0,1), \\
x(0)=0, \quad x(1)=\operatorname{ax}(\eta),
\end{array}\right.
$$

where $0<a<1,0<\eta<1$.

Step 2. We discuss the existence of a $C^{1}[0,1]$ solution of (2.11).

Now Lemma 2.2 guarantees that for each $h \in L^{1}[0,1]$, the linear problem

$$
\left\{\begin{array}{l}
-x^{\prime \prime}(t)+\lambda x=h, \quad t \in(0,1), \\
x(0)=0, \quad x(1)=\operatorname{ax}(\eta)
\end{array}\right.
$$

has an unique $C[0,1]$ solution

$$
v(t)=\int_{0}^{1} G(t, s) h(s) d s+\frac{e_{1}(t)}{e_{1}(1)-a e_{1}(\eta)} a \int_{0}^{1} G(\eta, s) h(s) d s, s \in[0,1] .
$$

For $x \in C^{1}[0,1]$, define

$$
(F x)(t)=g\left(t, x(t), x^{\prime}(t)\right)+\lambda \chi(t, x(t)), t \in[0,1]
$$

and

$$
(T x)(t)=\int_{0}^{1} G(t, s)(F x)(s) d s+\frac{e_{1}(t)}{e_{1}(1)-a e_{1}(\eta)} a \int_{0}^{1} G(\eta, s)(F x)(s) d s, s \in[0,1] .
$$

From (2.9) and (2.10), we have

$$
\left|g\left(t, x(t), x^{\prime}(t)\right)+\lambda \chi(t, x(t))\right| \leq \bar{N}(t)+\lambda \max \left\{\sup _{t \in[0,1]}|\alpha(t)|, \sup _{t \in[0,1]}|\beta(t)|\right\}, \quad \text { which }
$$

implies that the functions belonging to $\left\{(T x)(t): x \in C^{1}[0,1]\right\}$ and

$\left\{(T x)^{\prime}(t): x \in C^{1}[0,1]\right\}$ are bounded and equicontinuous. The Arzela-Ascoli Theorem guarantees that $T C^{1}[0,1]$ is relatively compact. The proof of the continuity of $T$ is standard. Using the Schauder's fixed point theorem, we assert that $T$ has at least one fixed point $x \in C^{1}[0,1]$.

Step 3. The solution $x$ of $(2.11)$ is such that $\alpha(t) \leq x(t) \leq \beta(t)$.

We prove that $x(t) \leq \beta(t)$ for $t \in[0,1]$ only. In fact, suppose that there exist a $t_{0} \in[0,1)$ such that $x\left(t_{0}\right)>\beta\left(t_{0}\right)$. Since $x(0)=0 \leq \beta(0), t_{0}>0$. Let $w(t)=x(t)-\beta(t), t \in[0,1]$. Then $w(0) \leq 0$ and $w\left(t_{0}\right)>0$.

Let $t^{*}=\sup \left\{t \mid w(s)>0, s \in\left[t_{0}, t\right]\right\}, \quad t_{*}=\inf \left\{t \mid w(s)>0, s \in\left[t, t_{0}\right]\right\}$.

It is obvious that $w(t)>0$ for all $t \in\left(t_{*}, t^{*}\right), w\left(t_{*}\right)=0$ and $w\left(t^{*}\right) \geq 0$. If $w\left(t^{*}\right)=0$, then there exists a $t^{\prime} \in\left(t_{*}, t^{*}\right)$ such that $w\left(t^{\prime}\right)=\max _{t \in\left[t_{*}, t^{*}\right]} w(t)$. If 
$w\left(t^{*}\right)>0$, obviously $t^{*}=1$ and $w(1)=x(1)-\beta(1)>0$. Since $w(\eta)=x(\eta)-\beta(\eta)=\frac{1}{a}(x(1)-\beta(1))=\frac{1}{a} w(1)>w(1)$, there exists $t^{\prime} \in\left(t_{*}, t^{*}\right)$ such that $w\left(t^{\prime}\right)=\max _{t \in\left[t_{*} t^{*}\right]} w(t)$ also. Hence, $w^{\prime}\left(t^{\prime}\right)=0$ (i.e., $\left.\beta^{\prime}\left(t^{\prime}\right)=x^{\prime}\left(t^{\prime}\right)\right)$ and $-w^{\prime \prime}\left(t^{\prime}\right) \geq 0$. On the other hand, since

$$
\begin{aligned}
-w^{\prime \prime}\left(t^{\prime}\right)= & \beta^{\prime \prime}\left(t^{\prime}\right)-x^{\prime \prime}\left(t^{\prime}\right) \\
\leq & -f\left(t^{\prime}, \beta\left(t^{\prime}\right), \beta\left(t^{\prime}\right)\right)+g\left(t^{\prime}, x\left(t^{\prime}\right), x^{\prime}(t)\right)+\lambda \chi\left(t^{\prime}, x\left(t^{\prime}\right)\right)-\lambda x\left(t^{\prime}\right) \\
= & -f\left(t^{\prime}, \beta\left(t^{\prime}\right), \beta^{\prime}\left(t^{\prime}\right)\right)+\max \left\{\min \left\{f\left(t^{\prime}, \beta\left(t^{\prime}\right), \beta^{\prime}(t)\right), \bar{N}(t)\right\},-\bar{N}(t)\right\} \\
& +\lambda \beta\left(t^{\prime}\right)-\lambda x\left(t^{\prime}\right) \\
= & -f\left(t^{\prime}, \beta\left(t^{\prime}\right), \beta^{\prime}\left(t^{\prime}\right)\right)+f\left(t^{\prime}, \beta\left(t^{\prime}\right), \beta^{\prime}\left(t^{\prime}\right)\right)+\lambda \beta\left(t^{\prime}\right)-\lambda x\left(t^{\prime}\right) \\
= & \lambda\left(\beta\left(t^{\prime}\right)-x\left(t^{\prime}\right)\right)<0 .
\end{aligned}
$$

This is a contradiction.

A similar argument holds to prove $x(t) \leq \beta(t)$ for all $t \in[0,1]$.

Hence, from (2.10), one know that $x$ satisfies that

$$
\left\{\begin{array}{l}
-x^{\prime \prime}(t)=g\left(t, x(t), x^{\prime}(t)\right)=\max \left\{\min \left\{f\left(t, x(t), x^{\prime}(t)\right), \bar{N}(t)\right\}, \quad t \in(0,1),\right. \\
x(0)=0, \quad x(1)=\operatorname{ax}(\eta) .
\end{array}\right.
$$

Step 4. The solution $x$ of (2.11) is such that $\left|x^{\prime}\right|_{\infty} \leq R$.

On the contrary, suppose that there is a $t^{\prime} \in(0,1)$ such that $\left|x^{\prime}\left(t^{\prime}\right)\right|>R$. Without loss of generality, we assume that $x^{\prime}\left(t^{\prime}\right)>R$. Since $x(0)=0$ and $x(1)=a x(\eta)$ with $0<a<1$, there is a $t_{0} \in(0,1)$ such that $x^{\prime}\left(t_{0}\right)=0$. Without loss of generality, we assume that $x^{\prime}(t)>0$ for all $\left(t^{\prime}, t_{0}\right)$. Observe that, for all $(t, x) \in D_{\alpha}^{\beta}, v \in \mathbb{R}$,

$$
\max \{\min \{f(t, x, v), \bar{N}(t)\},-\bar{N}(t)\} \leq \bar{\psi}(t) \bar{\phi}(|v|) .
$$

Then, from (2.12), one has

$$
\begin{aligned}
\int_{0}^{R} \frac{1}{\bar{\phi}(s)} d s & =\left|\int_{x^{\prime}\left(t_{0}\right)}^{x^{\prime}\left(t^{\prime}\right)} \frac{1}{\bar{\phi}(s)} d s\right|=\left|\int_{t^{\prime}}^{t_{0}} \frac{1}{\bar{\phi}\left(x^{\prime}(t)\right)} d x^{\prime}(t)\right| \\
& =\left|\int_{t^{\prime}}^{t_{0}} \frac{x^{\prime \prime}(t)}{\bar{\phi}\left(x^{\prime}(t)\right)} d t\right|=\left|\int_{t^{\prime}}^{t_{0}} \frac{g\left(t, x(t), x^{\prime}(t)\right)}{\bar{\phi}\left(x^{\prime}(t)\right)} d t\right| \\
& =\int_{t^{\prime}}^{t_{0}} \frac{\bar{\psi}(t) \bar{\phi}\left(x^{\prime}(t)\right)}{\bar{\phi}\left(x^{\prime}(t)\right)} d t=\int_{t^{\prime}}^{t_{0}} \bar{\psi}(t) d t=\|\bar{\psi}\|_{1} .
\end{aligned}
$$

This contradicts to (2.7).

Hence $\left|f\left(t, x(t), x^{\prime}(t)\right)\right| \leq \bar{N}(t)$, which together with $u \in[\alpha, \beta]$ guarantees that $g\left(t, x(t), x^{\prime}(t)\right)=f\left(t, x(t), x^{\prime}(t)\right), \forall t \in(0,1)$.

Step 5. We claim that $x(t)$ satisfies (2.1).

Since $\left|x^{\prime}\right|_{\infty} \leq R$ and $\alpha(t) \leq x(t) \leq \beta(t)$, by (2.8), (2.10) and (2.12), we have

$$
\left\{\begin{array}{l}
-x^{\prime \prime}(t)=\max \left\{\min \left\{f\left(t, x(t), x^{\prime}(t)\right), \bar{N}(t)\right\}=f\left(t, x(t), x^{\prime}(t)\right), \quad t \in(0,1),\right. \\
x(0)=0, \quad x(1)=\operatorname{ax}(\eta),
\end{array}\right.
$$

that is, $x(t)$ is a $C^{1}[0,1]$ solution of (2.1). The proof is complete.

Now we consider the following problem

$$
\left\{\begin{array}{l}
-x^{\prime \prime}(t)=f(t, x(t)), \quad t \in(0,1), \\
x(0)=0, x(\eta)=\operatorname{ax}(1),
\end{array}\right.
$$


where $\eta \in(0,1), \quad 0<a<1$ and $f \in[0,1] \times \mathbb{R} \times \mathbb{R}$.

Now we give the definitions of lower and upper solutions for problem (2.13).

Definition 2.2. (see [16]) A function $\alpha(t)$ is called a lower solution to the problem (2.13), if $\alpha(t) \in C[0,1] \cap C^{2}(0,1)$ and satisfies

$$
\left\{\begin{array}{l}
-\alpha^{\prime \prime}(t) \leq f(t, \alpha(t)), \quad t \in(0,1), \\
\alpha(0) \leq 0, \quad \alpha(1) \leq a \alpha(\eta) .
\end{array}\right.
$$

Upper solution is defined by reversing the above inequality signs in problem (2.14).

By Theorem 2.1, we have following result.

Corollary 2.1. Suppose that there exists a lower solution $\alpha(t)$ and an upper solution $\beta(t)$ of problem (2.1) such that $\alpha(t) \leq \beta(t), t \in[0,1]$ and there exists $F \in L^{1}[0,1]$ such that $|f(t, x)| \leq F(t)$ for all $(t, x) \in D_{\alpha}^{\beta}$. Then the problem (2.13) has at least one $\mathrm{C}[0,1]$ solution $x(t)$ satisfies $\alpha(t) \leq x(t) \leq \beta(t)$, $t \in[0,1]$.

Remark 2.1: This result can be found in [15]. So our theorem improves the works in the previous literature.

Lemma 2.3. Suppose that $f:(0,1) \times[0,+\infty) \rightarrow \mathbb{R}$ is a continuous functions such that $s^{-1} f(t, s)$ is strictly decreasing for $s>0$ at each $t \in(0,1)$. Let $w, v \in C[0,1] \cap C^{2}(0,1)$ satisfies:

1) $w^{\prime \prime}+f(t, w) \leq 0 \leq v^{\prime \prime}+f(t, v), \quad t \in(0,1)$;

2) $w, v>0, t \in(0,1)$ and $w(0) \geq v(0), w(1) \geq a w(\eta), \quad v(1) \leq a v(\eta)$;

3) $v^{\prime \prime} \in L^{1}[0,1]$.

Then $w(t) \geq v(t), \quad t \in[0,1]$.

Proof. By $v^{\prime \prime} \in L^{1}(0,1)$, we know that $v^{\prime}(0+)$ and $v^{\prime}(1-)$ exist and then $v \in C^{1}[0,1]$.

Suppose conversely $v(t) \not z w(t)$ on $[0,1]$. We may assume without loss of generality that there exists $t_{0} \in(0,1)$ such that $v\left(t_{0}\right)-w\left(t_{0}\right)=\max _{0 \leq t \leq 1}(v(t)-w(t))>0$. Let

$$
\begin{aligned}
& t_{*}=\inf \left\{t_{1} \mid 0 \leq t_{1}<t_{0}, v(t)>w(t), t \in\left(t_{1}, t_{0}\right)\right\}, \\
& t^{*}=\sup \left\{t_{2} \mid t_{0} \leq t_{2}<1, v(t)>w(t), t \in\left(t_{0}, t_{2}\right)\right\} .
\end{aligned}
$$

It's obvious that $0 \leq t_{*}<t^{*} \leq 1$ and $v\left(t_{*}\right)=w\left(t_{*}\right), v^{\prime}\left(t_{*}+\right) \geq D^{+} w\left(t_{*}+\right)$, where $D^{+}$denote Dini derivatives.

For $t^{*} \leq 1$, there are three cases.

1) $t^{*}<1$. Then $v\left(t^{*}\right)=w\left(t^{*}\right), v^{\prime}\left(t^{*}\right) \leq w^{\prime}\left(t^{*}\right), v(t)>w(t)$ for all $t \in\left(t_{*}, t^{*}\right)$.

2) $t^{*}=1$ and $v\left(t^{*}\right)=w\left(t^{*}\right), \quad v^{\prime}\left(t^{*}-\right) \leq D_{-} w\left(t^{*}-\right), \quad v(t)>w(t)$ for all $t \in\left(t_{*}, t^{*}\right)$, where $D_{-}$denotes Dini derivatives.

3) $t^{*}=1$ and $v\left(t^{*}\right)>w\left(t^{*}\right), v(t)>w(t)$ for all $t \in\left(t_{*}, t^{*}\right]$. Since $v(1)-w(1) \leq a(v(\eta)-w(\eta))<v(\eta)-w(\eta)$, then there is $t^{\prime} \in[\eta, 1]$ such that

$$
v\left(t^{\prime}\right)-w\left(t^{\prime}\right)>0,\left(v\left(t^{\prime}\right)-w\left(t^{\prime}\right)\right)^{\prime}<0 .
$$

Combining above (1), (2) and (3), there is a $t^{\prime}>t_{*}$ such that

$$
v\left(t_{*}\right)=w\left(t_{*}\right), v^{\prime}\left(t_{*}+\right) \geq D^{+} w\left(t_{*}+\right), v\left(t^{\prime}\right) \geq w\left(t^{\prime}\right), v^{\prime}\left(t^{\prime}-\right) \leq D_{-} w\left(t^{\prime}-\right),
$$


and

$$
v(t)>w(t), \forall t \in\left(t_{*}, t^{\prime}\right) .
$$

Let $y(t)=v^{\prime}(t) w(t)-w^{\prime}(t) v(t), \quad t \in\left(t_{*}, t^{\prime}\right)$. Then we have

$$
\lim _{t \rightarrow t_{*}+} \inf y(t) \geq 0 \geq \lim _{t \rightarrow t^{\prime}-} \sup y(t) \text {. }
$$

On the other hand,

$$
\begin{aligned}
y^{\prime}(t) & =w(t) v^{\prime \prime}(t)-w^{\prime \prime}(t) v(t) \\
& =-w(t) f(t, v(t)+v(t) f(t, w(t)) \\
& =w(t) v(t)\left(\frac{f(t, w(t))}{w(t)}-\frac{f(t, v(t))}{v(t)}\right) \\
& \geq 0
\end{aligned}
$$

for $t \in\left(t_{*}, t^{\prime}\right)$ and $y^{\prime}(t) \neq 0$ on $(\alpha, \beta)$. This implies $y\left(t^{\prime}\right)>y\left(t_{*}\right)$. This contradicts (2.15), so $v(t) \leq w(t)$. The proof is complete. $\square$

By analogous methods in [19], we establish the following maximal theorem, which can be used in the proof of the uniqueness of positive solutions.

Lemma 2.4. (maximal theorem) Suppose that $0<\eta<1$, and $F=\left\{x \in C[0,1] \cap C^{2}(0,1), x(1)-a x(\eta) \geq 0, x(0) \geq 0\right\}$, if $x(t) \in F$ such that $-x^{\prime \prime}(t) \geq 0$ for $t \in(0,1)$, then $x(t) \geq 0$ for $t \in[0,1]$.

\section{Proofs of Main Theorems}

In this section, we'll always assume that $f(t, x)=\lambda x^{p}-K(t) x^{-q}$.

\section{(A) The proof of Theorem 1.1.}

Proof.

1) We consider the problem

$$
\left\{\begin{array}{l}
-x^{\prime \prime}(t)+K(t) x^{-q}(t)=\lambda x^{p}(t), \quad t \in(0,1), \\
x(0)=0, \quad x(1)=a x(\eta),
\end{array}\right.
$$

where $0<q, p<1, K \in C[0,1], \quad K_{*}>0, \quad 0<a<1, \quad 0<\eta<1$ and $\lambda$ is a positive parameter.

In [19], when $f(t, x)$ is increasing in $x$, the problem

$$
\left\{\begin{array}{l}
-x^{\prime \prime}(t)=f(t, x), \quad t \in(0,1), \\
x(0)=\operatorname{ax}(\eta), \quad x(1)=0
\end{array}\right.
$$

has an unique $C^{1}[0,1]$ positive solution. From that, suppose that $x_{*}(t)$ is an unique $C^{1}[0,1]$ positive solution of the problem

$$
\left\{\begin{array}{l}
-x^{\prime \prime}(t)=x^{p}(t), \quad t \in(0,1), \\
x(0)=0, \quad x(1)=a x(\eta),
\end{array}\right.
$$

where $0<a<1, \quad 0<\eta<1$.

Set $\beta(t)=\lambda^{\frac{1}{1-p}} x_{*}(t)$. Then

$$
\begin{aligned}
-\beta^{\prime \prime}(t)+K(t) \beta^{-q}(t) & =\lambda^{\frac{1}{1-p}} x_{*}(t)+K(t) \lambda^{\frac{-q}{1-p}} x_{*}^{-q}(t) \\
& >\lambda^{\frac{1}{1-p}} x_{*}(t)+K_{*} \lambda^{\frac{-q}{1-p}} x_{*}^{-q}(t) \\
& >\lambda^{\frac{1}{1-p}} x_{*}^{p}(t),
\end{aligned}
$$




$$
\lambda \beta^{p}(t)=\lambda^{\frac{1}{1-p}} x_{*}^{p}(t)
$$

Thus $-\beta^{\prime \prime}(t)+K(t) \beta^{-q}(t)>\lambda \beta^{p}(t)$. Combining it with (3.1.2) we obtain

$$
\left\{\begin{array}{l}
-\beta^{\prime \prime}(t)+K(t) \beta^{-q}(t)>\lambda \beta^{p}(t), \quad t \in(0,1) \\
\beta(0)=0, \quad \beta(1)=a \beta(\eta)
\end{array}\right.
$$

Consequently, $\beta(t)$ is a upper solution of (3.1.1).

Set $\alpha(t)=M \varphi_{1}^{\frac{1}{1+q}}$, where $M$ is a positive constant and $\varphi_{1}$ is the first eigenfunction. Then

$$
\begin{aligned}
-\alpha^{\prime \prime}(t)+K(t) \alpha^{-q}(t) & =-\frac{2 M}{1+q} \varphi_{1}^{\frac{1-q}{1+q}}(t) \varphi_{1}{ }^{\prime \prime}(t)+\frac{K(t)}{M^{q} \varphi_{1}^{\frac{2 q}{1+q}}}-\frac{2(1-q) M\left|\varphi_{1}^{\prime}\right|^{2}}{(1+q)^{2} \varphi_{1}^{\frac{2 q}{1+q}}} \\
& =\frac{2 \lambda M}{1+q} \varphi_{1}^{\frac{2}{1+q}}+\frac{K(t)}{M^{q}} \varphi_{1}^{\frac{2 q}{1+q}}-\frac{2(1-q) M\left|\varphi_{1}\right|^{2}}{(1+q)^{2} \varphi_{1}^{\frac{2 q}{1+q}}} \\
& <\frac{2 \lambda M}{\varphi_{1}^{\frac{2}{1+q}}}+\frac{K^{*}}{M^{q}} \varphi_{1}^{\frac{2 q}{1+q}}-\frac{2(1-q) M\left|\varphi_{1}\right|^{2}}{(1+q)^{2} \varphi_{1}^{\frac{2 q}{1+q}}} .
\end{aligned}
$$

By Lemma 2.1 we have $\varphi_{1}(t)=\sin \left(\sqrt{\lambda_{1}} t\right), \varphi_{1}(t)=\sqrt{\lambda_{1}} \cos \left(\sqrt{\lambda_{1}} t\right)$. Thus there exists $\delta_{0}>0$ and $b \in(0,1)$ such that

$$
\begin{gathered}
\left|\varphi_{1}^{\prime}(t)\right|=\left|\sqrt{\lambda_{1}} \cos \left(\sqrt{\lambda_{1}} t\right)\right|>\delta_{0}, \quad t \in[0, b), \\
\left|\varphi_{1}(t)\right|=\left|\sin \left(\sqrt{\lambda_{1}} t\right)\right|>\delta_{0}, \quad t \in[b, 1] .
\end{gathered}
$$

a) On $[0, b)$, choosing $M \geq M_{1}=\left[\frac{(1+q)^{2} K^{*}}{2(1-q) \delta_{0}^{2}}\right]^{\frac{1}{1+q}}$, then we have

$$
\frac{K^{*}}{M^{q} \varphi_{1}^{\frac{2 q}{1+q}}} \leq \frac{\lambda_{1} M}{1+q} \varphi_{1}^{\frac{2}{1+q}} \text {. }
$$

b) On $[b, 1]$, choosing $M \geq M_{2}=\left[\frac{(1+q)^{2} K^{*}}{2(1-q) \delta_{0}^{2}}\right]^{\frac{1}{1+q}}$, then we have

$$
\frac{K^{*}}{M^{q} \varphi_{1}^{\frac{2 q}{1+q}}} \leq \frac{\lambda_{1} M}{1+q} \varphi_{1}^{\frac{2}{1+q}} .
$$

Fixing $M=\max \left\{M_{1}, M_{2}\right\}$, then

$$
-\alpha^{\prime \prime}(t)+K(t) \alpha^{-q}(t) \leq \frac{3 \lambda_{1} M}{1+q} \varphi_{1}^{\frac{2}{1+q}}
$$

and

$$
\lambda \alpha^{p}(t)=\lambda M^{p} \varphi_{1}^{\frac{2 q}{1+q}}
$$

Set $\lambda_{0}=\frac{3 M^{1-q}}{1+q}\left|\varphi_{1}\right|_{\infty}^{\frac{2-2 p}{1+q}}$. Then we have 


$$
\frac{3 M \lambda_{1}}{1+q} \varphi_{1}^{\frac{2}{1+q}}<\lambda M^{p} \varphi_{1}^{\frac{2 p}{1+q}}, \forall \lambda>\lambda_{0}
$$

Hence, $-\alpha^{\prime \prime}(t)+K(t) \alpha^{-q}(t)<\lambda \alpha^{p}(t), \quad \forall \lambda>\lambda_{0}$.

It follows from Lemma (2.1) that

$$
\alpha(0)=M \varphi_{1}^{\frac{2}{1+q}}(0)=0
$$

and

$$
\alpha(1)=M \varphi_{1}^{\frac{2}{1+q}}(1)=M\left[a \varphi_{1}(\eta)\right]^{\frac{2}{1+q}}=M a^{\frac{2}{1+q}} \varphi_{1}^{\frac{2}{1+q}}(\eta)<a M \varphi_{1}^{\frac{2}{1+q}}(\eta)=a \alpha(\eta) .
$$

Set $\lambda_{2}=\left(M\left|\frac{\varphi_{1}}{x_{*}}\right|_{\infty}\left|\varphi_{1}\right|_{\infty}^{\frac{1-q}{1+q}}\right)^{1-p}$. Then $\quad \alpha(t)=M \varphi_{1}^{\frac{2}{1+q}}(t) \leq \lambda^{\frac{1}{1-p}} x_{*}(t)=\beta(t)$ for all $\lambda>\lambda_{2}$. Thus we choose $\bar{\lambda}=\max \left\{\lambda_{0}, \lambda_{2}\right\}$ and $\lambda>\bar{\lambda}$, then $(\alpha(t), \beta(t))$ is a couple of upper and lower solutions of (3.1.1).

We choose $F(t)=\lambda \beta^{p}+K^{*} \beta^{-q}$, then $|f(t, x)| \leq F(t)$ for all $(t, x) \in D_{\alpha}^{\beta}$. It's easy to see that $F(t) \in L^{1}[0,1]$. From Corollary 2.1, the problem (3.1.1) has at least one $C[0,1]$ positive solution $x(t)$ satisfying $\alpha(t) \leq x(t) \leq \beta(t)$ for $\lambda>\bar{\lambda}$.

2) (Existence of the maximal solution) We observe the problem

$$
\left\{\begin{array}{l}
-x^{\prime \prime}(t)=\lambda x^{p}(t), \quad t \in(0,1) \\
x(0)=0, \quad x(1)=\operatorname{ax}(\eta)
\end{array}\right.
$$

From [19], we note the unique solution of (3.1.3) is $w_{\lambda}(t)$ for any $\lambda>0$. In (1) we obtained the solution $x_{\lambda}(t)$ of (3.1.1) then we have

$$
w_{\lambda}{ }^{\prime \prime}(t)+\lambda w_{\lambda}^{p}(t)=0<x_{\lambda} "(t)+\lambda x_{\lambda}^{p}(t)
$$

and $x^{-1} f(t, x)=\lambda x_{\lambda}^{p-1}(t)$ is decreasing in $x$. Noting that $x_{\lambda}(t) \in L^{1}[0,1]$ by (1). From Lemma 2.3, we have $x_{\lambda}(t) \leq w_{\lambda}(t)$.

Let $\Omega_{j}=\left[\frac{1}{i_{0}+j}, 1\right), j=1,2, \cdots$ and $w_{j}(t)$ be the solution of

$$
\left\{\begin{array}{l}
-x^{\prime \prime}(t)+K(t) w_{j-1}^{-q}(t)=\lambda w_{j-1}^{p}(t), \quad t \in \Omega_{j}, \\
x(t)=w_{j-1}(t), \quad t \in\left[0, \frac{1}{i_{0}+j}\right), \\
x(1)=\operatorname{ax}(\eta)
\end{array}\right.
$$

for $j=1,2, \cdots$, with $w_{0}(t)=w_{\lambda}(t)$ defined in (3.1.3). Let $x_{\lambda}(t)$ be a solution of (3.1.1).

In (3.1.4), letting $j=1$ we have

$$
\left\{\begin{array}{l}
-w_{1}^{\prime \prime}(t)+K(t) w_{\lambda}^{-q}(t)=\lambda w_{\lambda}^{p}(t), \quad t \in \Omega_{1}, \\
w_{1}(t)=w_{\lambda}(t), t \in\left[0, \frac{1}{i_{0}+j}\right), \\
w_{1}(1)=a w_{1}(\eta) .
\end{array}\right.
$$

Combining (3.1.5) with (3.1.3) we have $w_{1} "(t)-w_{\lambda} "(t) \geq 0$ for $t \in \Omega_{1}$. By maximum principle, we have $w_{1}(t) \leq w_{0}(t)=w_{\lambda}(t)$. Similarly, we can obtain that $w_{j+1}(t) \leq w_{j}(t) \leq w_{\lambda}(t)$. 
Furthermore, we observe problem (3.1.1)

$$
\left\{\begin{array}{l}
-x^{\prime \prime}(t)+K(t) x^{-q}(t)=\lambda x^{p}(t), \quad t \in(0,1), \\
x(0)=0, \quad x(1)=a x(\eta) .
\end{array}\right.
$$

Combining it with (3.1.5) we have

$$
-w_{1}{ }^{\prime \prime}(t)+x_{\lambda}{ }^{\prime \prime}(t)+K(t)\left(w_{\lambda}^{-q}(t)-x_{\lambda}^{-q}(t)\right)=\lambda\left(w_{\lambda}^{p}(t)-x_{\lambda}^{p}(t)\right) \geq 0,
$$

thus $x_{\lambda}{ }^{\prime \prime}(t)-w_{1} "(t) \geq 0$ for $t \in \Omega_{1}$. It's easy to verify that $x_{\lambda}(t) \leq w_{1}(t)$ for $t \in[0,1]$ by maximum principle. By similar method we can obtain $x_{\lambda}(t) \leq w_{j+1}(t) \leq w_{j}(t) \leq w_{\lambda}(t)$ for $t \in[0,1]$.

Furthermore, we have $\left\{w_{j}(t)\right\}_{j \in N}$ is bounded from below by $x_{\lambda}(t)$.

Because $w_{j}(t)$ is a solution to (3.1.3),

$$
\begin{aligned}
-w_{j} "(t) & =\lambda w_{j-1}^{p}(t)-K(t) w_{j-1}^{-q}(t) \\
& \leq \lambda w_{j-1}^{p}(t)-K_{*} w_{j-1}^{-q}(t) \\
& \leq\left[\lambda w_{j-1}^{p+q}(t)-K_{*}\right] w_{j-1}^{-q}(t) \\
& \leq\left[\lambda w_{j-1}^{p+q}(t)-K_{*}\right] w_{j}^{-q}(t) .
\end{aligned}
$$

Suppose that $t_{0} \in(0,1), w_{j}\left(t_{0}\right)=\max _{0 \leq t \leq 1} w_{j}(t)$, then $w_{j}^{\prime}\left(t_{0}\right)=0$ and $w_{j}(t)$ is increasing on $\left(t, t_{0}\right)$. By integration of $-w_{j}$ " $(t)$ from $t$ to $t_{0}$, we have

$$
\int_{t}^{t_{0}}-w_{j} "(s) d s \leq \int_{t}^{t_{0}}\left[\lambda w_{j-1}^{p+q}(s)-K_{*}\right] w_{j}^{-q}(s) d s .
$$

So $w_{j}{ }^{\prime}(t) w_{j}^{q}(t) \leq \lambda w_{j-1}^{p+q}\left(t_{0}\right)-K_{*}$. Similarly, by integration of $-w_{j}{ }^{\prime \prime}(t)$ from $t_{0}$ to $t$, we can obtain $\left|w_{j}^{\prime}(t) w_{j}(t)\right| \leq \lambda w_{j-1}^{p+q}\left(t_{0}\right)-K_{*}$. For giving $t_{1}, t_{2} \in[0,1]$, we have

$$
\int_{t_{1}}^{t_{2}} w_{j}^{\prime}(s) w_{j}^{q}(s) d s \leq \int_{t_{1}}^{t_{2}}\left|w_{j}^{\prime}(s) w_{j}^{q}(s)\right| d s \leq \int_{t_{1}}^{t_{2}}\left[\lambda w_{j-1}^{p+q}\left(t_{0}\right)-K_{*}\right] d s .
$$

We can find $K$ large such that $\left|\lambda w_{j-1}^{p+q}\left(t_{0}\right)-K_{*}\right|<K$. Then

$$
\int_{t_{1}}^{t_{2}} w_{j}^{\prime}(s) w_{j}^{q}(s) d s \leq K\left|t_{2}-t_{1}\right|,\left|w_{j}^{q+1}\left(t_{2}\right)-w_{j}^{q+1}\left(t_{1}\right)\right| \leq K\left|t_{2}-t_{1}\right| .
$$

We define an operator $I(w)=w^{q+1}$, then $I^{-1}(w)=w^{\frac{1}{q+1}}$. It follows from (3.1.4) that $\left\{I\left(w_{j}(t)\right)\right\}_{j \in N}$ is a uniformly bounded and equicontinuous functions in $[0,1]$. Obviously, $I^{-1}$ is uniformly continuous in a bounded and closed domain $\Omega$, i.e., for all $\varepsilon>0$, there exists a $\delta>0$ such that when $w_{1}$, $w_{2} \in \Omega \quad, \quad\left|w_{1}-w_{2}\right|<\delta$, we have $\left|I^{-1}\left(w_{1}\right)-I^{-1}\left(w_{2}\right)\right|<\varepsilon$. Since $0<w_{j}(t)<w_{0}(t)$, there exists a $M>0$ such that $w_{j}(t) \in(0, M]$. From (3.1.4), for the above $\delta>0$, there exists $\delta^{\prime}>0$ such that when $\left|t_{1}-t_{2}\right|<\delta^{\prime}$, we have $\left|w_{j}^{q+1}\left(t_{2}\right)-w_{j}^{q+1}\left(t_{1}\right)\right|<\delta$.

Therefore, for all $\varepsilon>0$, there exists $\delta^{\prime}>0$ such that when $\left|t_{1}-t_{2}\right|<\delta^{\prime}$, we have

$$
\left|w_{j}\left(t_{2}\right)-w_{j}\left(t_{1}\right)\right|=\left|I^{-1}\left(w_{j}^{q+1}\left(t_{2}\right)\right)-I^{-1}\left(w_{j}^{q+1}\left(t_{1}\right)\right)\right|<\varepsilon .
$$

Thus $\left\{w_{j}(t)\right\}_{j \in N}$ is equicontinuous. Using Arzela-Ascoli theorem, there exists a subsequence $\left\{w_{j_{k}}(t)\right\}_{j_{k} \in\{i\}}$ such that $\lim _{j_{k} \rightarrow+\infty} w_{j_{k}}(t)=\bar{x}_{\lambda}(t)$. Without loss of generality, we assume that 


$$
\lim _{j \rightarrow+\infty} w_{j}(t)=\bar{x}_{\lambda}(t), \quad t \in[0,1] .
$$

In the following, we shall show that $\bar{x}_{\lambda}(t)$ is a $C[0,1]$ positive solution of (3.1.1).

Fixing $t \in(0,1)\left(t \neq \frac{1}{2}\right)$, then $w_{j}(t)$ can be stated

$$
w_{j}(t)=w_{j}\left(\frac{1}{2}\right)+w_{j}^{\prime}\left(\frac{1}{2}\right)\left(t-\frac{1}{2}\right)+\int_{\frac{1}{2}}^{t}(s-t)\left[K(s) w_{j-1}^{-q}(s)-\lambda w_{j-1}^{p}(s)\right] d s .
$$

Fixing $j \in N$, by Lagrange mean value theorem, there exists $t_{n} \in\left(\frac{1}{2}, 1\right)$ such that $x_{\lambda}(1)-w_{j}\left(\frac{1}{2}\right) \leq w_{j}(1)-w_{j}\left(\frac{1}{2}\right)=w_{j^{\prime}}\left(t_{n}\right)\left(1-\frac{1}{2}\right)<w_{0}(1)$.

So there exists $M_{1}>0$ such that $\left|w_{j^{\prime}}\left(t_{n}\right)\right|<2 M_{1}$. Since $\left\{w_{j}(t)\right\}_{j \in N}$ is bounded in [0,1], we may assume that $m<w_{j}(t)<M_{2}, t \in\left[\frac{1}{2}, t_{n}\right]$,

$$
\begin{aligned}
\mid \int_{\frac{1}{2}}^{t_{n}}-w_{j}^{\prime \prime}(s) d s & |=| \int_{\frac{1}{2}}^{t_{n}}\left[\lambda w_{j-1}^{p}(s)-K(s) w_{j-1}^{-q}(s)\right] d s \mid \\
& \leq\left|\int_{\frac{1}{2}}^{t_{n}}\left[\lambda w_{j-1}^{p}(s)-K_{*} w_{j-1}^{-q}(s)\right] d s\right| \\
& \leq \lambda M^{p}-K_{*} m^{-q} .
\end{aligned}
$$

Thus

$$
\left|w_{j}^{\prime}\left(\frac{1}{2}\right)\right|-\left|w_{j}^{\prime}\left(t_{n}\right)\right| \leq\left|w_{j}^{\prime}\left(\frac{1}{2}\right)-w_{j}^{\prime}\left(t_{n}\right)\right| \leq \lambda M_{2}^{p}-K^{*} m^{-q}
$$

i.e.,

$$
\left|w_{j}^{\prime}\left(\frac{1}{2}\right)\right| \leq 2 M_{1}+\lambda M_{2}^{p}-K^{*} m^{-q} .
$$

Thus both $\left\{w_{j}{ }^{\prime}\left(\frac{1}{2}\right)\right\}_{j \in N}$ and $\left\{w_{j}\left(\frac{1}{2}\right)\right\}_{j \in N}$ are bounded. Then they all have a convergence subsequence. Without loss of generality, we note the subsequences are $\left\{w_{j}\left(\frac{1}{2}\right)\right\}_{j \in N}$ and $\left\{w_{j}{ }^{\prime}\left(\frac{1}{2}\right)\right\}_{j \in N}$. And fixing $j \in N$, we assume $\lim _{j \rightarrow \infty} w_{j}{ }^{\prime}\left(\frac{1}{2}\right)=r_{0}$.

In equation (3.1.6), letting $j \rightarrow \infty$ we have

$$
\bar{x}_{\lambda}(t)=\bar{x}_{\lambda}\left(\frac{1}{2}\right)+r_{0}\left(t-\frac{1}{2}\right)+\int_{\frac{1}{2}}^{t}(s-t)\left[K(s) \bar{x}_{\lambda}^{-q}(s)-\lambda \bar{x}_{\lambda}^{p}(s)\right] d s
$$

for $t \in(0,1)$, i.e., $-\bar{x}_{\lambda}{ }^{\prime \prime}(t)+K(t) \bar{x}_{\lambda}^{-q}(t)=\lambda \bar{x}_{\lambda}^{p}(t)$. Therefore $\bar{x}_{\lambda}(t)$ is a $\mathrm{C}[0,1]$ positive solution of (3.1.1). Therefore $\bar{x}_{\lambda}(t)$ is the maximal solution of (3.1.1).

Next we shall verify the dependence on $\lambda$ of maximal solution $\bar{x}_{\lambda}(t)$.

Let $H=\{\mu>0:$ (3.1.1) has a $\mathrm{C}[0,1]$ positive solution with $\lambda=\mu\}$.

Obviously, by (1), $H \neq \varnothing$. Let $\lambda_{1} \in H$. and $\bar{x}_{\lambda}(t)$ be the corresponding maximal solution of (3.1.1) for $\lambda=\lambda_{1}$. Then for any $\lambda_{2}>\lambda_{1}>\bar{\lambda}$, $\bar{x}_{\lambda_{1}}{ }^{\prime \prime}(t)+\lambda_{1} \bar{x}_{\lambda_{1}}^{p}(t) \geq 0, t \in(0,1)$. By Lemma $(2.3), \bar{x}_{\lambda_{1}}(t) \leq w_{\lambda_{2}}(t)$ in [0,1]. Just replacing $x_{\lambda}(t)$ by $\bar{x}_{\lambda_{1}}(t)$ in above proof. We can easily find that

$$
\left\{\begin{array}{l}
-\bar{x}_{\lambda_{1}}^{\prime \prime}(t)+K(t) \bar{x}_{\lambda_{1}}^{-q}(t)=\lambda_{1} \bar{x}_{\lambda_{1}}^{p} \leq \lambda_{2} \bar{x}_{\lambda_{1}}^{p}, \quad t \in(0,1), \\
-w_{\lambda_{2}}^{\prime \prime}(t)+K(t) w_{\lambda_{2}}^{-q}(t) \geq \lambda_{2} w_{\lambda_{2}}^{p}(t) .
\end{array}\right.
$$


Combining it with boundary conditions, we can obtain that $\left(\bar{x}_{\lambda_{1}}(t), w_{\lambda_{2}}(t)\right)$ is a couple of lower and upper solutions of (3.1.1) for $\lambda=\lambda_{2}>\lambda_{1}$. One can be prove that there is a solution $x_{\lambda_{2}}(t)$ of (3.1.1) with $\lambda=\lambda_{2}$ such that

$$
\bar{x}_{\lambda_{1}}(t) \leq x_{\lambda_{2}}(t) \leq w_{\lambda_{2}}(t)
$$

Therefore $\lambda_{2} \in H$. Moreover, by (ii), for any $\lambda_{2}>\lambda_{1} \geq \bar{\lambda}, \bar{x}_{\lambda_{2}}(t) \geq \bar{x}_{\lambda_{1}}(t)$.

This completes the proof of Theorem 1.1.

(B) The proof of Theorem 1.2.

Proof. 1) We consider the problem

$$
\left\{\begin{array}{l}
-x^{\prime \prime}(t)+K(t) x^{-q}(t)=\lambda x^{p}(t), \quad t \in(0,1) \\
x(0)=0, \quad x(1)=\operatorname{ax}(\eta)
\end{array}\right.
$$

where $q>0,0<p<1, K(t) \in C[0,1], K^{*}<0,0<a<1,0<\eta<1$ and $\lambda$ is a positive parameter.

Now we consider an approximate problem of (3.2.1) as follows

$$
\left\{\begin{array}{l}
-x^{\prime \prime}(t)+K(t) x^{-q}(t)=\lambda x^{p}(t), \quad t \in(0,1), \\
x(0)=\frac{1}{n}, \quad x(1)=\operatorname{ax}(\eta)+\frac{1}{n},
\end{array}\right.
$$

where $0<a<1, \quad 0<\eta<1, \quad n \geq 1$.

Let $\varepsilon$ very small. We'll verify that $\alpha_{n}(t)=\varepsilon \varphi_{1}(t)+\frac{1}{n}$ is a lower solution of (3.2.2). Indeed, when $n$ is big enough, we can obtain that $\varepsilon \varphi_{1}(t)+\frac{1}{n}$ is close to 0. Since $\lambda_{1} \in\left(0, \sqrt{\frac{\pi}{2}}\right) \quad($ see $[6])$, we can deduce

$$
\begin{aligned}
& -\alpha_{n}{ }^{\prime}(t)+K(t) \alpha_{n}^{-q}(t)-\lambda \alpha_{n}^{p}(t) \\
& =\lambda_{1} \varepsilon \varphi_{1}(t)+K(t)\left(\varepsilon \varphi_{1}(t)+\frac{1}{n}\right)^{-q}-\lambda\left(\varepsilon \varphi_{1}(t)+\frac{1}{n}\right)^{p} \\
& <\lambda_{1} \varepsilon \varphi_{1}(t)-\lambda\left(\varepsilon \varphi_{1}(t)+\frac{1}{n}\right)^{p} \\
& <\varepsilon \varphi_{1}(t)\left[\lambda_{1}-\lambda\left(\varepsilon \varphi_{1}(t)+\frac{1}{n}\right)^{p-1}\right] \\
& <0, \quad \alpha_{n}(0)-\frac{1}{n}=\varepsilon \varphi_{1}(0)=0
\end{aligned}
$$

and $\alpha_{n}(1)-\left[a \alpha_{n}(\eta)+\frac{1}{n}\right]=\varepsilon a \varphi_{1}(\eta)+\frac{1}{n}-a \varepsilon \varphi_{1}(\eta)-\frac{a}{n}-\frac{1}{n}<0$, which imply that $\alpha_{n}(t)$ is a lower solutions of (3.2.2).

In the following, we'll construct an upper solution of (3.2.2). Let

$$
\beta(t)=-M t^{2}+(M+a M) t+M,
$$

where $M$ is big enough for $M>\left\{(2 \lambda)^{\frac{1}{1-p}}, \frac{1}{n(1-a)}\right\}$. We can obtain 


$$
\begin{aligned}
&-\beta^{\prime \prime}(t)+K(t) \beta^{-q}(t)=2 M+K(t)\left[-M t^{2}+(M+a M) t+M\right]^{-q} \\
&>2 M+K_{*} M^{-q} \\
&>M, \\
& \lambda \beta^{p}(t)=\lambda\left[-M t^{2}+(M+a M) t+M\right]^{p} \\
&<\lambda\left[\frac{M(1+a)^{2}}{4}+M\right]^{p} \\
&<\lambda(2 M)^{p}, \\
&-\beta^{\prime \prime}(t)+K(t) \beta^{-q}(t) \geq \lambda \beta^{p}(t), \\
& \beta(1)-\left(a \beta(\eta)+\frac{1}{n}\right)=(a+1) M-a\left[-M \eta^{2}+(M+a M) \eta+M\right]-\frac{1}{n} \\
&>(a+1) M-2 a M-\frac{1}{n} \\
&= M-a M-\frac{1}{n} \\
&> 0
\end{aligned}
$$

and $\beta(0)-\frac{1}{n}=M-\frac{1}{n}>0$. It's easy to see that $\beta(t)$ is na upper solution of (3.2.2).

Choosing $F_{n}(t)=\lambda \beta^{p}-K_{*} \alpha_{n}^{-q}$, then $|f(t, x)| \leq F_{n}(t)$, for all $(t, x) \in D_{\alpha_{n}}^{\beta}$. It's easy to verify that $F_{n}(t) \in L^{1}[0,1]$. Because that $\varepsilon$ is small and $n$ is big enough, $\alpha_{n}(t) \leq \beta(t)$. From Corollary 2.1, $\left(\alpha_{n}(t), \beta(t)\right)$ is a couple of upper and lower solutions of (3.2.2). And for all $n \in N,(3.2 .2)$ has at least one $\mathrm{C}[0,1]$ positive solution $x_{n}(t)$ such that $\alpha_{n}(t) \leq x_{n}(t) \leq \beta(t)$.

In the following, we shall obtain a result as follows, there exists a subsequence $\left\{x_{n_{k}}(t)\right\}$ and $x(t)$ such that $\lim _{n_{k} \rightarrow \infty} x_{n_{k}}(t)=x(t)$.

Since $\beta(t) \in C[0,1] \cap C^{2}(0,1), \beta(t)$ is bounded. Therefore $\left\{x_{n}(t)\right\}_{n \in N}$ is a uniformly bounded sequence of functions in $[0,1]$. Because $x_{n}(t)$ is a $\mathrm{C}[0,1]$ positive solution of (3.2.2), $x_{n}(t)$ satisfies

$$
\begin{aligned}
-x_{n}{ }^{\prime \prime}(t) & =\lambda x_{n}^{p}(t)-K(t) x_{n}^{-q}(t) \\
& \leq \lambda x_{n}^{p}(t)-K_{*} x_{n}^{-q}(t) \\
& \leq\left[\lambda x_{n}^{p+q}(t)-K_{*}\right] x_{n}^{-q}(t) .
\end{aligned}
$$

Suppose that $t_{0} \in(0,1), \quad x_{n}\left(t_{0}\right)=\max x_{n}(t)$, then $x_{n}{ }^{\prime}\left(t_{0}\right)=0$ and $x_{n}(t)$ is increasing on $\left(t, t_{0}\right)$. By integration of ${ }^{0, t \leq 1}-x_{n}$ " $(t)$ from $t$ to $t_{0}$, we have

$$
\int_{t}^{t_{0}}-x_{n} "(s) d s \leq \int_{t}^{t_{0}}\left[\lambda x_{n}^{p+q}(s)-K_{*}\right] x_{n}^{-q}(s) d s .
$$

So $x_{n}{ }^{\prime}(t) \leq \frac{1}{x_{n}^{q}(t)}\left[\lambda x_{n}^{p+q}\left(t_{0}\right)-K_{*}\right]$. We can find a $K>0$ such that $x_{n}{ }^{\prime}(t) x_{n}^{q}(t) \leq K$. And by integration of $-x_{n}(t)$ from $t_{0}$ to $t$, we have

$$
\int_{t}^{t_{0}}-x_{n} "(s) d s \leq \int_{t}^{t_{0}}\left[\lambda x_{n}^{p+q}(s)-K_{*}\right] x_{n}^{-q}(s) d s .
$$

So $-x_{n}{ }^{\prime}(t) \leq \frac{1}{x_{n}^{q}(t)}\left[\lambda x_{n}^{p+q}\left(t_{0}\right)-K_{*}\right]$. For above $K$, we have $\left|-x_{n}{ }^{\prime}(t) x_{n}^{q}(t)\right| \leq K$, 
i.e., $\left|x_{n}{ }^{\prime}(t) x_{n}^{q}(t)\right| \leq K$.

For giving $t_{1}, t_{2} \in[0,1]$, we have

$$
\int_{t_{1}}^{t_{2}} x_{n}{ }^{\prime}(s) x_{n}^{q}(s) d s \leq \int_{t_{1}}^{t_{2}}\left|x_{n}{ }^{\prime}(s) x_{n}^{q}(s)\right| d s \leq \int_{t_{1}}^{t_{2}} K d s .
$$

Then $\int_{t_{1}}^{t_{2}} x_{n}{ }^{\prime}(s) x_{n}^{q}(s) d s \leq K\left|t_{2}-t_{1}\right|$. The above inequality can be rewritten as

$$
\left|\int_{x_{n}\left(t_{1}\right)}^{x_{n}\left(t_{2}\right)} x_{n}^{q}(s) d x_{n}(s)\right| \leq K\left|t_{2}-t_{1}\right|, \quad\left|x_{n}^{q+1}\left(t_{2}\right)-x_{n}^{q+1}\left(t_{1}\right)\right| \leq K\left|t_{2}-t_{1}\right| .
$$

We now define an operator $I(x)=x^{q+1}$, then $I^{-1}(x)=x^{\frac{1}{q+1}}$. It follows from (3.2.3) that $\left\{I\left(x_{n}(t)\right)\right\}_{n \in N}$ is a uniformly bounded and equicontinuous functions in $[0,1]$. Obviously, $I^{-1}$ is uniformly continuous in a bounded and closed domain $\Omega$, i.e., for all $\varepsilon>0$, there exists a $\delta>0$ such that $\left|I^{-1}\left(x_{1}\right)-I^{-1}\left(x_{2}\right)\right|<\varepsilon$ for $\left|x_{1}-x_{2}\right|<\delta, \quad x_{1}, x_{2} \in \Omega$. Since $0<x_{n}(t)<\beta(t)$, there exists a $M>0$ such that $x_{n}(t) \in(0, M]$. From (3.2.3), for the above $\delta>0$, there exists $\delta^{\prime}>0$ such that $\left|x_{n}^{q+1}\left(t_{2}\right)-x_{n}^{q+1}\left(t_{1}\right)\right|<\delta$ for $\left|t_{1}-t_{2}\right|<\delta^{\prime}$.

Therefore, for all $\varepsilon>0$, there exists $\delta^{\prime}>0$ such that

$$
\left|x_{n}\left(t_{2}\right)-x_{n}\left(t_{1}\right)\right|=\left|I^{-1}\left(x_{n}^{q+1}\left(t_{2}\right)\right)-I^{-1}\left(x_{n}^{q+1}\left(t_{1}\right)\right)\right|<\varepsilon
$$

for $\left|t_{1}-t_{2}\right|<\delta^{\prime}$. Consequently, $\left\{x_{n}(t)\right\}_{n \in N}$ is equicontinuous. Using Arzela-Ascoli theorem, there exists a subsequence $\left\{x_{n_{k}}(t)\right\}$ such that $\lim _{n_{k} \rightarrow+\infty} x_{n_{k}}(t)=x(t)$. Without loss of generality, we assume that

$$
\lim _{n \rightarrow+\infty} x_{n}(t)=x(t), \quad t \in[0,1] .
$$

In the following, we shall show that $x(t)$ is a $\mathrm{C}[0,1]$ positive solution of (3.2.1). Fixing $t \in(0,1)\left(t \neq \frac{1}{2}\right), x_{n}(t)$ can be stated

$$
x_{n}(t)=x_{n}\left(\frac{1}{2}\right)+x_{n}{ }^{\prime}\left(\frac{1}{2}\right)\left(t-\frac{1}{2}\right)+\int_{\frac{1}{2}}^{t}(s-t)\left[K(s) x_{n}^{-q}(s)-\lambda x_{n}^{p}(s)\right] d s .
$$

Fixing $n \in N$, by Lagrange mean value theorem, there exists $t_{n} \in\left(\frac{1}{2}, 1\right)$ such that $\alpha_{n}(1)-x_{n}\left(\frac{1}{2}\right) \leq x_{n}(1)-x_{n}\left(\frac{1}{2}\right)=x_{n}{ }^{\prime}\left(t_{n}\right)\left(1-\frac{1}{2}\right) \leq \beta(1)$.

So there exists $M_{1}>0$ such that $\left|x_{n}{ }^{\prime}\left(t_{n}\right)\right| \leq 2 M_{1}$. Since $\left\{x_{n}(t)\right\}_{n \in N}$ is bounded in $[0,1]$, we may assume that $m \leq x_{n}(t) \leq M_{2}, t \in\left[\frac{1}{2}, t_{n}\right]$.

$$
\left|\int_{\frac{1}{2}}^{t_{n}}-x_{n}^{\prime \prime}(s) d s\right|=\left|\int_{\frac{1}{2}}^{t_{n}}\left[\lambda x_{n}^{p}(s)-K(s) x_{n}^{-q}(s)\right] d s\right| .
$$

We can obtain

$$
\left|-x_{n}{ }^{\prime}\left(t_{n}\right)+x_{n}{ }^{\prime}\left(\frac{1}{2}\right)\right| \leq \lambda M_{2}^{p}-K_{*} M_{2}^{-q} \text { and }\left|x_{n}{ }^{\prime}\left(\frac{1}{2}\right)\right| \leq 2 M_{1}+\lambda M_{2}^{p}-K_{*} M_{2}^{-q} \text {. }
$$

Therefore both $\left\{x_{n}\left(\frac{1}{2}\right)\right\}_{n \in N}$ and $\left\{x_{n}{ }^{\prime}\left(\frac{1}{2}\right)\right\}_{n \in N}$ are bounded. They all have a convergence subsequence. Without loss of generality, we note the subsequences are $\left\{x_{n}\left(\frac{1}{2}\right)\right\}_{n \in N}$ and $\left\{x_{n}{ }^{\prime}\left(\frac{1}{2}\right)\right\}_{n \in N}$. And fixing $n \in N$, we assume $\lim _{n \rightarrow \infty} x_{n}{ }^{\prime}\left(\frac{1}{2}\right)=r_{0}$. 
From (3.2.5), letting $n \rightarrow \infty$, we obtain

$$
x(t)=x\left(\frac{1}{2}\right)+r_{0}\left(t-\frac{1}{2}\right)+\int_{\frac{1}{2}}^{t}(s-t)\left[K(s) x^{-q}(s)-\lambda x^{p}(s)\right] d s .
$$

By derivation twice of $x(t)$, we have

$$
-x^{\prime \prime}(t)+K(t) x^{-q}(t)=\lambda x^{p}(t) .
$$

Combining it with (3.2.4), we can obtain that $x(t)$ is a $\mathrm{C}[0,1]$ positive solution of (3.2.1).

2) We study the uniqueness of $C^{1}[0,1]$ positive solution of problem (3.2.1).

Let $F(t)=\lambda \beta^{p}-K_{*}\left(\varepsilon \varphi_{1}\right)^{-q}$. Obviously, when $0<q<1, F(t)$ is integrable over $(0,1)$. Since $\left|x^{\prime \prime}(t)\right| \leq F(t), x(t)$ is absolutely integrable over $(0,1)$. Then both $x^{\prime}(0+)$ and $x^{\prime}(1-)$ exist, i.e., $x(t) \in C^{1}[0,1]$.

Suppose conversely that $x_{1}(t), x_{2}(t)$ are two $C^{1}[0,1]$ positive solutions of the problem (3.2.1), $x_{1}(t) \neq x_{2}(t)$ on $[0,1]$. We may assume without loss of generality that there exists $t^{*} \in(0,1)$ such that

$$
\begin{aligned}
& x_{2}\left(t^{*}\right)-x_{1}\left(t^{*}\right)=\max _{0 \leq t \leq 1}\left(x_{2}(t)-x_{1}(t)\right)>0 . \text { Let } \\
& \alpha=\inf \left\{t_{1} \mid 0 \leq t_{1}<t^{*}, x_{2}(t)>x_{1}(t), t \in\left(t_{1}, t^{*}\right)\right\}, \\
& \quad \beta=\sup \left\{t_{2} \mid t^{*} \leq t_{2}<1, x_{2}(t)>x_{1}(t), t \in\left(t^{*}, t_{2}\right)\right\} .
\end{aligned}
$$

It's obvious that $0 \leq \alpha<\beta \leq 1$ and

$$
\begin{aligned}
& x_{1}(\alpha)=x_{2}(\alpha), x_{1}{ }^{\prime}(\alpha) \leq x_{2}{ }^{\prime}(\alpha), x_{1}(\beta) \leq x_{2}(\beta), \\
& x_{1}{ }^{\prime}(\beta+) \geq x_{2}{ }^{\prime}(\beta+), x_{1}(t)<x_{2}(t), t \in(\alpha, \beta) .
\end{aligned}
$$

Let $y(t)=x_{1}(t) x_{2}{ }^{\prime}(t)-x_{2}(t) x_{1}{ }^{\prime}(t), t \in(\alpha, \beta)$. Then we have

$$
\lim _{t \rightarrow \alpha+} \inf y(t) \geq 0 \geq \lim _{t \rightarrow \beta+} \sup y(t) .
$$

On the other hand,

$$
\begin{aligned}
y^{\prime}(t) & =x_{1} x_{2}^{\prime \prime}-x_{2} x_{1}^{\prime \prime} \\
& =x_{1}\left(K x_{2}^{-q}-\lambda x_{2}^{p}\right)+x_{2}\left(\lambda x_{1}^{p}-K x_{1}^{-q}\right) \\
& =K x_{1} x_{2}^{-q}-\lambda x_{1} x_{2}^{p}+\lambda x_{1}^{p} x_{2}-K x_{1}^{-q} x_{2} \\
& =K x_{1} x_{2}\left(x_{2}^{-q-1}-x_{1}^{-q-1}\right)+\lambda x_{1} x_{2}\left(x_{1}^{p-1}-x_{2}^{p-1}\right) \\
& \geq 0
\end{aligned}
$$

for $t \in(\alpha, \beta)$ and $y^{\prime}(t) \not \equiv 0$ on $(\alpha, \beta)$. This implies $y(\beta-)>y(\alpha+)$, contradicts (3.2.6), so $x_{1}(t) \equiv x_{2}(t)$. Thus the $C^{1}[0,1]$ positive solution of (3.2.6) is unique.

3) We assume that $0<\lambda_{1}<\lambda_{2}$ and $x_{\lambda_{1}}(t), x_{\lambda_{2}}(t)$ are the corresponding unique $C^{1}[0,1]$ positive solutions to (3.2.1). Obviously, $x_{\lambda_{1}}{ }^{\prime \prime}(t) \in L^{1}[0,1]$. In (3.2.1), $f(t, x)=\lambda x^{p}(t)-K(t) x^{-q}(t)$ is continuous.

Since $p, q \in(0,1), K_{*}<0$, it's easy to see that $x^{-1} f(t, x)=\lambda x^{p-1}(t)-K(t) x^{-q-1}(t)$ is decreasing for $x>0$ at each $t \in[0,1]$.

$$
x_{\lambda_{2}}{ }^{\prime \prime}(t)-K(t) x_{\lambda_{2}}^{-q}(t)+\lambda_{2} x_{\lambda_{2}}^{p}(t)=0<x_{\lambda_{1}}{ }^{\prime \prime}(t)-K(t) x_{\lambda_{1}}^{-q}(t)+\lambda_{2} x_{\lambda_{1}}^{p}(t)
$$

for $t \in(0,1), \quad x_{\lambda_{2}}(0) \geq x_{\lambda_{1}}(0), \quad x_{\lambda_{2}}(1) \geq a x_{\lambda_{2}}(\eta)$ and $x_{\lambda_{1}}(1) \geq a x_{\lambda_{1}}(\eta)$. There- 
fore, by Lemma 2.3,

$$
x_{\lambda_{1}(t)} \leq x_{\lambda_{2}}(t), \quad t \in[0,1] .
$$

So $x(t)$ is increasing with respect to $\lambda$.

This completes the proof of Theorem 1.2.

(C) The proof of Theorem 1.3.

Proof.

1) We consider the problem

$$
\left\{\begin{array}{l}
-x "(t)+K(t) x^{-q}(t)=\lambda x^{p}(t), \quad t \in(0,1), \\
x(0)=0, \quad x(1)=a x(\eta)
\end{array}\right.
$$

where $0<p, q<1, K(t) \in C[0,1], \quad K_{*}<0<K^{*}, \quad 0<a<1, \quad 0<\eta<1$ and $\lambda$ is a positive parameter.

Since $K^{*}>0>K_{*}$, then by Theorem 1.1, there exists a $\lambda^{*}>0$, such that for $\lambda>\lambda^{*}$, the problem

$$
\left\{\begin{array}{l}
-v^{\prime \prime}(t)+K^{*} v^{-q}(t)=\lambda v^{p}(t), \quad t \in(0,1), \\
v(0)=0, \quad v(1)=a v(\eta)
\end{array}\right.
$$

has a maximal solution $v_{\lambda}(t)$. Let $v_{k}(t)=v_{\lambda}(t)+\frac{1}{k}$. We observe that

$$
\begin{aligned}
&-v_{k}^{\prime \prime}(t)+K(t) v_{k}^{-q}(t)=-v_{\lambda}^{\prime \prime}(t)+K(t)\left(v_{\lambda}+\frac{1}{k}\right)^{-q} \\
&=\lambda v_{\lambda}^{p}(t)-K^{*} v_{\lambda}^{-q}(t)+K\left(v_{\lambda}+\frac{1}{k}\right)^{-q} \\
&<\lambda v_{\lambda}^{p}(t)+K^{*}\left(v_{\lambda}+\frac{1}{k}\right)^{-q}-K^{*} v_{\lambda}^{-q}(t) \\
&<\lambda v_{\lambda}^{p}(t) . \\
& \lambda v_{k}^{p}(t)=\lambda\left(v_{\lambda}+\frac{1}{k}\right)>\lambda v_{\lambda}^{p}(t), \\
& v_{k}(0)=v_{\lambda}(0)+\frac{1}{k}=\frac{1}{k} \text { and } \quad v_{k}(1)=v_{\lambda}(1)+\frac{1}{k}=a v_{\lambda}(\eta)+\frac{1}{k} \leq a v_{k}(\eta)+\frac{1}{k} .
\end{aligned}
$$

Consequently, $v_{k}(t)=v_{\lambda}(t)+\frac{1}{k}$ is a lower solution of $P_{k}(\lambda)$ :

$$
\left\{\begin{array}{l}
-x^{\prime \prime}(t)+K(t) x^{-q}(t)=\lambda x^{p}(t), \quad t \in(0,1), \\
x(0)=\frac{1}{k}, \quad x(1)=a x(\eta)+\frac{1}{k} .
\end{array}\right.
$$

On the other hand, the problem

$$
\left\{\begin{array}{l}
-w^{\prime \prime}(t)+K_{*} w^{-q}(t)=\lambda w^{p}(t), \quad t \in(0,1), \\
w(0)=\frac{1}{k}, \quad w(1)=a w(\eta)
\end{array}\right.
$$

has a solution $w_{k}(t)$ for any $k \in N$. Then

$$
\begin{aligned}
-w_{k} "(t)+K(t) w_{k}^{-q}(t) & =\lambda w_{k}^{p}(t)-K_{*} w_{k}^{-q}(t)+K(t) w_{k}^{-q}(t) \\
& >\lambda w_{k}^{p}(t)-K_{*}(t) w_{k}^{-q}(t)+K_{*} w_{k}^{-q}(t), \\
& =\lambda w_{k}^{p}(t) .
\end{aligned}
$$

So we have 


$$
\left\{\begin{array}{l}
-w_{k} "(t)+K(t) w_{k}^{-q}(t)>\lambda w_{k}^{p}(t), \quad t \in(0,1) \\
w_{k}(0)=\frac{1}{k} \\
w_{k}(1)=a w_{k}(\eta) .
\end{array}\right.
$$

Therefore, $w_{k}(t)$ is an upper solution of $P_{k}(\lambda)$. Since $w_{k}{ }^{\prime \prime}(t)+\lambda w_{k}^{p}(t) \leq 0 \leq v_{k} "(t)+\lambda v_{k}^{p}(t), \quad w(0) \geq v(0), \quad w(1) \geq a w(\eta), \quad v(1) \leq a v(\eta)$, $v^{\prime \prime}(t) \in L^{1}[0,1]$ and $x^{-1} f(t, x)=\lambda x^{p-1}(t)$ is decreasing in $x$, by Lemma 2.3,

$$
v_{k}(t) \leq w_{k}(t), \quad t \in[0,1] .
$$

Obviously, there exists a minimal solution $x_{\lambda}^{(1)}(t)$ of $P_{1}(\lambda)$, satisfying $v_{1}(t) \leq x_{\lambda}^{(1)}(t) \leq w_{1}(t)$. Similarly, taking $x_{\lambda}^{(1)}(t)$ and $v_{2}(t)$ as a couple of lower and upper solutions for $P_{2}(\lambda)$, we conclude that there exists a minimal solution $x_{\lambda}^{(2)}(t)$ of $P_{2}(\lambda)$ such that

$$
v_{2}(t) \leq x_{\lambda}^{(2)}(t) \leq x_{\lambda}^{(1)}(t) .
$$

Repeating the above arguments, we obtain a sequence $\left\{x_{\lambda}^{(k)}(t)\right\}_{k \in N}$ which is decreasing in $k$. Therefore, similar to the proof of Theorem $1.2(1)$, we obtain a solution $x_{\lambda}(t)=\lim _{k \rightarrow \infty} x_{\lambda}^{(k)}(t)$, and $v_{\lambda}(t) \leq x_{\lambda}(t) \leq w_{1}(t)$.

2) (Dependence on $\lambda$ ) Let $\lambda^{*}<\lambda_{1}<\lambda_{2}, x_{\lambda_{1}}(t)$ and $x_{\lambda_{2}}(t)$ be the corresponding solutions of (3.3.1) for $\lambda=\lambda_{1}$ and $\lambda_{2}$ which we obtained in (1). We observe that

$$
\left\{\begin{array}{l}
-\left(x_{\lambda_{2}}^{(k)}(t)\right)^{\prime \prime}+K_{*}\left(x_{\lambda_{2}}^{(k)}(t)\right)^{-q}=\lambda_{2}\left(x_{\lambda_{2}}^{(k)}(t)\right)^{p} \geq \lambda_{1}\left(x_{\lambda_{2}}^{(k)}(t)\right)^{p}, \quad t \in(0,1), \\
x_{\lambda_{2}}^{(k)}(0)=0, \quad x_{\lambda_{2}}^{(k)}(1)=a x_{\lambda_{2}}^{(k)}(\eta),
\end{array}\right.
$$

$x_{\lambda_{2}}^{(k)}(t)$ is an upper solution of $P_{k}\left(\lambda_{1}\right)$, and

$$
x_{\lambda_{2}}^{(k)}(t) \geq v_{\lambda_{2}}(t)+\frac{1}{k} \geq v_{\lambda_{1}}(t)+\frac{1}{k}, t \in[0,1] .
$$

Therefore $x_{\lambda_{2}}^{(k)}(t) \geq x_{\lambda_{1}}^{(k)}(t)$, since $x_{\lambda_{1}}^{(k)}(t)$ is a minimal solution of $P_{k}\left(\lambda_{1}\right)$ which satisfies $x_{\lambda_{1}}^{(k)}(t) \geq v_{\lambda_{1}}(t)+\frac{1}{k}$. Therefore we must have $x_{\lambda_{1}}(t) \leq x_{\lambda_{2}}(t)$.

Thus Theorem 1.3 is true.

\section{Funding}

This work is supported by the National Natural Science Foundation of China (61603226) and the Fund of Natural Science of Shandong Province (ZR2018MA022).

\section{Availability of Data and Materials}

Not applicable.

\section{Conflicts of Interest}

The authors declare that they have no competing interests. 


\section{Authors' Contributions}

All authors contributed equally to the writing of this paper. All authors read and approved the final manuscript.

\section{References}

[1] Ilin, V.A. and Moiseev, E.I. (1987) Nonlocal Boundary Value of the First Kind for a Sturm-Liouville Operator in Its Differential and Finite Difference Aspects. Differential Equations, 23, 803-810.

[2] Ilin, V.A. and Moiseev, E.I. (1987) Nonlocal Boundary Value of the Second Kind for Sturm-Liouville Operator. Differential Equations, 23, 979-987.

[3] Du, X. and Zhao, Z. (2008) Existence and Uniqueness of Positive Solutions to a Class of Singular M-Point Boundary Value Problems. Applied Mathematics and Computation, 198, 487-493. https://doi.org/10.1016/j.amc.2007.08.080

[4] Gupta, C.P. (1998) A Generalized Multi-Point Boundary Value Problem for Second Order Ordinary Differential Equations. Applied Mathematics and Computation, 89, 133-146. https://doi.org/10.1016/S0096-3003(97)81653-0

[5] Ma, R. and O'Regan, D. (2005) Solvability of Singular Second Order M-Point Boundary Value Problems. Journal of Mathematical Analysis and Applications, 301, 124-134. https://doi.org/10.1016/j.jmaa.2004.07.009

[6] Rynne, B.P. (2007) Spectral Properties and Nodal Solutions for Second-Order, M-Point Boundary Value Problems. Nonlinear Analysis, 67, 3318-3327.

https://doi.org/10.1016/j.na.2006.10.014

[7] Rynne, B.P. (2008) Second-Order, Three-Point, Boundary Value Problems with Jumping Non-Linearities. Nonlinear Analysis, 68, 3294-3306. https://doi.org/10.1016/j.na.2007.03.023

[8] Brno, A.L. and Granada, P.T. (2003) Onatwo-Point Boundary Value Problem for Second Order Singular Equations Czechoslovak. Mathematical Journal, 53, 19-43.

[9] De Coster, C. and Habets, P. (1996) Upper and Lower Solutions in the Theory of Ode Boundary Value Problems: Classical and Recent Results. Nonlinear Analysis and Boundary Value Problems for Ordinary Differential Equations, 371, 1-78.

[10] De Coster, C. and Habets, P. (2004) The Lower and Upper Solutions Method for Boundary Value Problems. Handbook of Differential Equations, Ordinary Differential Equations, 1, 69-160.

[11] Habets, P. and Zanolin, F. (1994) Upper and Lower Solutions for a Generalized Emden-Fowler Equation. Journal of Mathematical Analysis and Applications, 181, 684-700. https://doi.org/10.1006/jmaa.1994.1052

[12] O’Regan, D., Yan, B. and Agarwal, R.P. (2007) Solutions in Weighted Spaces of Singular Boundary Value Problems on the Half-Line. Journal of Computational and Applied Mathematics, 205, 751-763. https://doi.org/10.1016/j.cam.2006.02.055

[13] O’Regan, D. and Agarwal, R.P. (2000) Singular Problems: An Upper and Lower Solution Approach. Journal of Mathematical Analysis and Applications, 251, 230-250. https://doi.org/10.1006/jmaa.2000.7043

[14] Sankar, L., Sasi, S. and Shivaji, R. (2013) Semipositone Problems with Falling Zeros on Exterior Domains. Journal of Mathematical Analysis and Applications, 401, 146-153. https://doi.org/10.1016/j.jmaa.2012.11.031

[15] Wei, Z. (2008) Positive Solutions of Sublinear Singular Three-Point Boundary Value Problems. Acta Mathematica Scientia, 28, 174-182. 
[16] Yan, B., O'Regan, D. and Agarwal, R.P. (2006) Unbounded Solutions for Singular Boundary Value Problems on the Semi-Infinite Interval: Upper and Lower Solutions and Multiplicity. Journal of Computational and Applied Mathematics, 197, 365-386. https://doi.org/10.1016/j.cam.2005.11.010

[17] Yan, B. and Zhang, M. (2015) Positive Solutions of Two-Point Boundary Value Problems for Monge-Ampre Equations. Journal of Function Spaces, Article ID: 620251, 8 p. https://doi.org/10.1155/2015/620251

[18] Zhang, Z. (1995) On a Drichlet Problem with a Singular Nonlinearity. Journal of Mathematical Analysis and Applications, 194, 103-113.

[19] Du, X. and Zhao, Z. (2007) A Necessary and Sufficient Condition for the Existence of Positive Solutions to Singular Sunlinear Three-Point Boundary Value Problems. Applied Mathematics and Computation, 186, 404-413. https://doi.org/10.1016/j.amc.2006.07.120

[20] Biles, D.C., Robinson, M.P. and Spraker., J.S. (2002) A Generalization of the Lane-Emden Equation. Journal of Mathematical Analysis and Applications, 273, 654-666. https://doi.org/10.1016/S0022-247X(02)00296-2

[21] Callegari, A. and Nashman, A. (1980) A Nonlinear Singular Boundary Value Problem in the Theory of Pseudoplastic Fluids. SIAM J. Appl. Math., 38, 275-281. https://doi.org/10.1137/0138024

[22] Cohen, D.S. and Keller, H.B. (1967) Some Positive Problems Suggested by Nonlinear Heat Generators. J. Math.Mech., 16, 1361-1376.

[23] Diaz, J.I., Morel, J.M. and Oswald, L. (1987) An Elliptic Equation with Singular Nonlinearity. Comm. Partial Differential Equations, 12, 1333-1344.

[24] Fulks, W. and Maybee, J.S. (1960) A Singular Nonlinear Equation. Osaka Math. J., 12, 1-19.

[25] Shi, J. and Yao, M. (1998) On a Singular Nonlinear Semilinear Elliptic Problem. Proceedings of the Royal Society of Edinburgh, 12, 1389-1401. https://doi.org/10.1017/S0308210500027384

[26] Ma, R.Y. and Castaneda, N. (2001) Existence of Solutions of Nonlinear M-Point Boundary Value Problems. Journal of Mathematical Analysis and Applications, 256, 556-567. https://doi.org/10.1006/jmaa.2000.7320 